\title{
Fiberoptic bronchoscopy for the rapid diagnosis of smear-negative pulmonary tuberculosis
}

\author{
Jung Ar Shin', Yoon Soo Chang ${ }^{1}$, Tae Hoon Kim², Hyung Jung Kim', Chul Min Ahn¹ and Min Kwang Byun ${ }^{\text {* }}$
}

\begin{abstract}
Background: This study was aimed to investigate the diagnostic value of fiberoptic bronchoscopy (FOB) with chest high-resolution computed tomography (HRCT) for the rapid diagnosis of active pulmonary tuberculosis (PTB) in patients suspected of PTB but found to have a negative sputum acid-fast bacilli (AFB) smear.

Methods: We evaluated the diagnostic accuracy of results from FOB and HRCT in 126 patients at Gangnam Severance Hospital (Seoul, Korea) who were suspected of having PTB.

Results: Of 126 patients who had negative sputum AFB smears but were suspected of having PTB, 54 patients were confirmed as having active PTB. Hemoptysis was negatively correlated with active PTB. Tree-in-bud appearance on HRCT was significantly associated with active PTB. The sensitivity, specificity, positive predictive value (PPV), and negative predictive value (NPV) of FOB alone was 75.9\%, 97.2\%, 95.3\%, and 84.3\%, respectively, for the rapid diagnosis of active PTB. The combination of FOB and HRCT improved the sensitivity to $96.3 \%$ and the NPV to $96.2 \%$

Conclusions: FOB is a useful tool in the rapid diagnosis of active PTB with a high sensitivity, specificity, PPV and NPV in sputum smear-negative PTB-suspected patients. HRCT improves the sensitivity of FOB when used in combination with FOB in sputum smear-negative patients suspected of having PTB.
\end{abstract}

\section{Background}

Tuberculosis (TB) is a major health problem around the world, with an approximate incidence of 9.4 million (8.9-9.9 million) and with about 1.7 million TB-related deaths in 2009 [1,2]. The global strategy to control TB is prompt diagnosis, notification, and successful treatment of patients with active, transmissible disease. Early diagnosis of active pulmonary tuberculosis (PTB) is critical for TB control.

Unfortunately, diagnosis of active PTB is often delayed because fewer than half of these patients have a positive sputum smear (29-39\% in 2009 in Korea) [1,3], and isolation of Mycobacterium tuberculosis (MTB) takes a long time. Acid-fast bacilli (AFB) smears of respiratory specimens (at least two or more specimens) are important for the prompt diagnosis of PTB, but AFB smears have poor sensitivity (30-70\%) despite high specificity (98-99\%).

\footnotetext{
* Correspondence: littmann@yuhs.ac

'Department of Internal Medicine, Yonsei University College of Medicine, Gangnam Severance Hospital, 211 Eonju-ro, Gangnam-gu Seoul 135-720, South Korea

Full list of author information is available at the end of the article
}

Mycobacterial cultures are more sensitive than AFB smears (80-85\%), but culture results usually require $3-8$ weeks [4]. The diagnosis of $\mathrm{TB}$ and the decision to start treatment against sputum smear-negative TB is usually dependent on clinical features, but $20 \%$ of PTB patients are completely asymptomatic whereas $42-86 \%$ of PTB patients may be symptomatic. Sputum smear-negative PTB patients are especially likely to show no or mild respiratory symptoms and systemic manifestations [5].

Chest $\mathrm{x}$-ray is another method often used in PTB screening, but in sputum smear-negative TB, many cases showed atypical $x$-ray patterns or normal findings [6]. In addition, this method has the limitation of a high false positive rate due to previous PTB infection in an intermediate or high TB burden country. To assist in the diagnosis of sputum smear-negative PTB and latent TB infection (LTBI), clinicians use the tuberculin skin test (TST). However, the TST is limited in application due to cross-reactivity with non-tuberculous mycobacteria (NTM) species and Bacillus Calmette-Geurin (BCG) vaccine strains, and its sensitivity is affected by both malnutrition and immunosuppression [7]. 
Chest high-resolution computed tomography (HRCT) provides information about the extent and distribution of PTB and can be a great help in identifying its activity. In sputum smear-negative cases, HRCT is superior to chest $\mathrm{x}$-ray in the diagnosis of PTB and in the determination of its extent and distribution. Further, it confirms the presumptive diagnosis of active PTB and allows antituberculosis treatment to commence more quickly. Despite some disadvantages such as high cost and radiation exposure, HRCT is widely used when traditional methods have failed to diagnose PTB in sputum smearnegative patients. Typical radiological patterns of PTB reactivation such as upper lobe involvement or cavity formation are rarely observed in sputum smear-negative cases due to the smaller burden of mycobacterium [5]; therefore, HRCT alone is limited in diagnosing PTB in sputum smear-negative patients.

Fiberoptic bronchoscopy (FOB) can provide alternative respiratory specimens for diagnosis $[8,9]$, especially from specific sites that are suspected by radiological testing for involvement of PTB when sputum expectoration has repeatedly failed because sputum is absent [10]. FOB is also more useful in the diagnosis of endobronchial TB, which can be seen as normal in HRCT, and FOB may be superior in the differential diagnosis of tuberculosis with other commonly encountered diseases such as pneumonia or lung cancer [11]. Despite the fact that it is more aggressive and relatively expensive, FOB is considered useful for the diagnosis of sputum smear-negative PTB because of these advantages. Results of bronchial washing specimens with AFB smears or MTB polymerase chain reaction (PCR) and tissue specimens from bronchoscopic or transbronchial biopsies are usually received within one week, thus enabling rapid diagnosis before the availability of confirmation from sputum cultures of sputum smear-negative PTB patients.

The aim of this study was to evaluate the diagnostic value of FOB with HRCT in the diagnosis of active PTB in patients suspected of PTB but presenting with a negative sputum smear in an intermediate TB burden country.

\section{Methods}

\section{Study setting and subjects}

We retrospectively reviewed the clinical records and FOB and HRCT results of all patients with suspected PTB who visited the pulmonary clinic of Gangnam Severance Hospital, Seoul, Korea from January 2009 to December 2010.

Inclusion criteria were (1) age of at least 18 years with clinical or radiographic suspicion of active PTB, (2) negative AFB smear results of 2 or more sputum pairs or failure to expectorate sputum (in other words, could not expectorate sputum voluntarily, did not submit any sputum specimen) and (3) having received both FOB and HRCT within 1 month due to suspected PTB before initiation of anti-TB treatment. When FOB was performed, a bronchial washing from the affected lung was acquired for AFB smear, mycobacterial culture and MTB-PCR for all patients. A bronchoscopic biopsy was performed on patients with an endobronchial lesion. Exclusion criteria were (1) AFB smear-positive patients, (2) patients with clinically diagnosed PTB according to clinical and radiological tuberculosis findings who showed no clinical improvement with empirical antibiotics but did show clinical and radiological improvement with anti-tuberculosis medication, and (3) patients with inconclusive diagnoses due to loss of follow-up.

From January 2009 to December 2010, 182 patients who were suspected of having PTB visited our clinic and underwent FOB and chest CT. HRCT was performed with contrast $\mathrm{CT}$, simultaneously. After excluding smear-positive PTB patients and patients with other exclusion criteria, we evaluated the diagnostic accuracy of FOB and HRCT results in 126 patients. The recorded clinical information on these patients included age, gender, cough, sputum, fever, hemoptysis, chest pain, and weight loss.

Bronchoscopic procedure is performed according to our institute's infection regulation and instruction guideline. Inspectors wear N95 masks, goggles and gown during procedure, bronchosopy room is equipped with negative pressure isolation and air disinfection system, Bronchoscopy is performed according to manufacturer's guidelines plus scope surveillance.

\section{Diagnostic definition}

Active PTB was confirmed when (1) MTB was cultured or (2) a caseating granuloma was found in the lung tissue by bronchoscopic biopsy or transthoracic needle biopsy, and when the PTB showed appropriate response to treatment. In cases diagnosed by pathology, active PTB was confirmed only if MTB tissue culture or tissue MTB-PCR was positive, for excluding NTM or other granulomatous diseases. A final diagnosis of 'non-TB' was accepted when an alternative diagnosis was reached.

A rapid diagnosis of sputum smear-negative PTB by FOB was defined as a diagnosis of PTB through methods that yielded results within 1 week: (1) a positive AFB smear, (2) positive MTB-PCR, or (3) a caseating granuloma upon biopsy.

CT findings were reviewed by two experienced thoracic radiologists, without clinical information or final diagnosis in each case. CT findings were described as having more than one of the following characteristics: (1) consolidation, (2) cavities, (3) centrilobular air space nodules, (4) tree-in-bud appearance and (5) hilar and/or mediastinal lymph node enlargement. After reviewing 
the CT findings independently, the two radiologists met, discussed and reached a final diagnosis by consensus.

The definition of an immunocompromised condition included the following: (1) having a diagnosis of diabetes mellitus, (2) undergoing chemotherapy for an underlying malignancy at the time of TST and QuantiFERON ${ }^{\circledR}$-TB Gold InTube (QFT-IT, Cellestis Ltd; Carnegie, Australia) testing, (3) having had received either a solid organ transplant or bone marrow transplant, (4) having a diagnosis of end-stage renal disease and on renal replacement therapy, (5) having a diagnosis of advanced liver cirrhosis with Child-Pugh class C, (6) being seropositive for human immunodeficiency virus, or (7) undergoing daily administration of systemic corticosteroids (at least $15 \mathrm{mg}$ prednisone per day for more than 1 month) or combination therapy with low dose corticosteroids and other immunosuppressants including azathioprine, mycophenolate, methotrexate, cyclosporine, or cyclophosphamide.

For the combination of FOB and HRCT, the results of the combination were considered positive when at least one test was positive.

\section{Analysis}

Data were analyzed with SPSS statistical software (version 18.0; SPSS; Chicago, IL, USA). Univariate comparisons between active $\mathrm{PTB}$ and non-TB patients were performed using Fisher's exact test for categorical variables and the Mann-Whitney test for continuous variables, where appropriate. Associations of clinical and radiological parameters with active PTB diagnosis were analyzed using univariate or multivariate logistic regression modeling. For multivariate analysis, variables were incorporated into the model in a stepwise manner. All tests of significance were two-sided and a P value $<0.05$ was considered statistically significant. Odds ratios (ORs) and 95\% confidence intervals (95\% CIs) were calculated. Sensitivity, specificity, positive predictive value (PPV) and negative predictive value (NPV) for the diagnosis of active PTB disease were calculated for each diagnostic test.

\section{Ethical issues}

This study was approved by the Institutional Review Board of the Gangnam Severance Hospital, Yonsei University College of Medicine (IRB No: 3-2011-0118).

\section{Results}

\section{Demographic characteristics}

Of the 145 patients with suspected sputum smearnegative $\mathrm{TB}$, five patients were excluded due to clinically diagnosed $\mathrm{PTB}$, and 14 patients were excluded due to inconclusive diagnoses (Figure 1).

Baseline characteristics of the non-excluded 126 patients are summarized in Table 1. Active PTB was diagnosed in 54 patients (42.9\%), of which 48 (88.9\%) had positive $\mathrm{TB}$ cultures and $6(11.1 \%)$ cases were

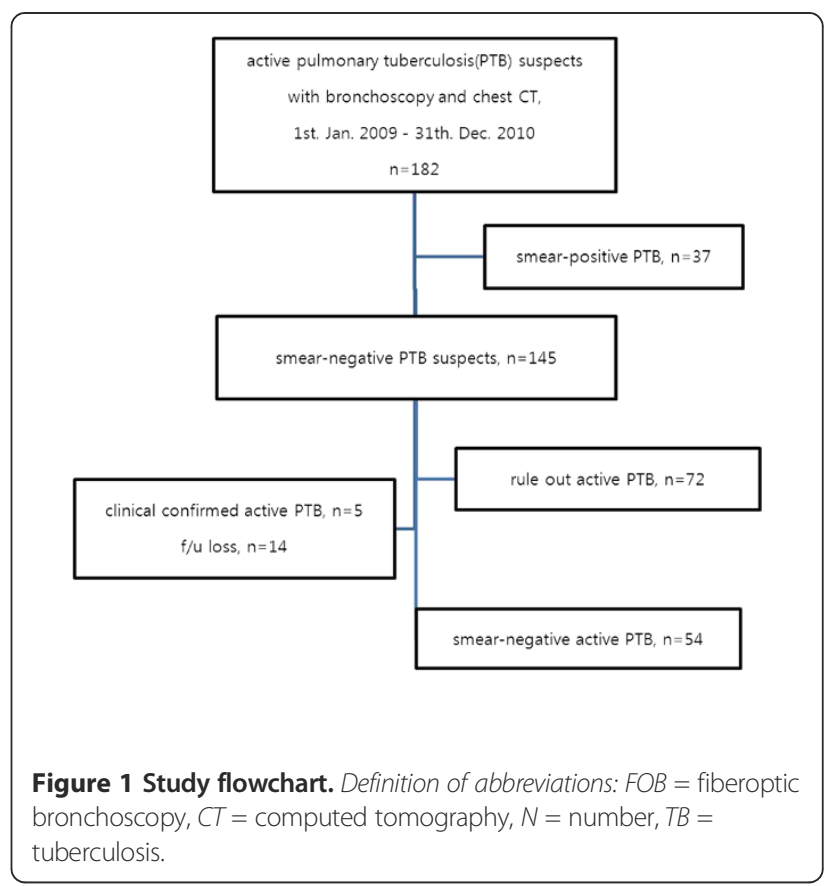

confirmed by pathology and MTB-PCR. Of 48 cultureconfirmed cases, 28 were culture positive by both sputum and bronchial washing specimens, 6 by sputum only, and 14 by bronchial washing specimens only.

Alternative (non-TB) diagnoses were made for 72 patients (34 pneumonia, 5 NTM colonization, 15 sequelae of a previous TB infection, 12 lung cancers, and 6 interstitial lung disease).

The mean patient age was $51.0 \pm 18.2$ years, and the mean age of the non-TB group was higher than that of the TB group $(55.4 \pm 16.6$ years vs. $45.3 \pm 18.8$ years, respectively, $\mathrm{P}=0.002$ ). Immunocompromised conditions were found in 11 patients ( 6 diabetes, 2 chemotherapy, 2 hematologic malignancies, and 1 systemic corticosteroid use). There was no difference between the TB and nonTB groups in terms of immunocompromised status, but previous TB history was significantly different between the TB and non-TB groups $(\mathrm{P}=0.014$, Table 1$)$.

\section{Clinical characteristics and HRCT findings in sputum smear-negative TB suspects}

Chest $x$-rays showed a higher positive rate in the TB group than in the non-TB group $(53.7 \%$ vs. $20.8 \%$, respectively, $\mathrm{P}<0.001$ ), and QFT-IT also showed significantly higher positive rate in the TB group than in the non-TB group $(87.0 \%$ vs. $46.4 \%$, respectively, $\mathrm{P}=0.009)$ (Table 1). Only hemoptysis $(\mathrm{P}=0.009$, OR $=0.30[0.12-$ $0.76,95 \% \mathrm{CI}]$ ) was negatively correlated with active PTB in sputum smear-negative suspects (Table 2).

Fifty-three patients were suspected of having active PTB by HRCT, and 38 (71.7\%) were correctly diagnosed with active PTB. Of the 73 patients suspected to be non- 
Table 1 Demographic and clinical characteristics of all sputum smear-negative TB suspects

\begin{tabular}{|c|c|c|c|c|}
\hline Characteristic & $\begin{array}{l}\text { All suspects } \\
\qquad N=126\end{array}$ & $\begin{array}{c}\text { TB } \\
\mathrm{N}=54\end{array}$ & $\begin{array}{l}\text { Non-TB } \\
\mathrm{N}=72\end{array}$ & P-value \\
\hline Age, years (mean $\pm S D$ ) & $51.0 \pm 18.2$ & $45.3 \pm 18.8$ & $55.4 \pm 16.6$ & 0.002 \\
\hline Male, n (\%) & $68(54.0)$ & $26(48.1)$ & $42(58.3)$ & 0.256 \\
\hline Previous TB history, n (\%) & $33(26.2)$ & $8(14.8)$ & $25(34.7)$ & 0.014 \\
\hline Immunocompromised patient, n (\%) & $11(8.7)$ & $4(7.4)$ & $7(9.7)$ & 0.649 \\
\hline CXR positivity, n (\%) & $44(34.9)$ & $29(53.7)$ & $15(20.8)$ & $<0.001$ \\
\hline QFT-IT positivity", n (\%) & $33 / 51(64.7)$ & $20 / 23(87.0)$ & $13 / 28(46.4)$ & 0.009 \\
\hline Active pulmonary TB, n (\%) & $54(42.9)$ & $54(100.0)$ & & \\
\hline Culture confirmed, $\mathrm{n}(\%)^{\dagger}$ & & $48(88.9)$ & & \\
\hline Biopsy confirmed, n (\%) & & $6(11.1)$ & & \\
\hline
\end{tabular}

Definition of abbreviations: $T B=$ tuberculosis, $S D=$ standard deviation, $n=$ number, $C X R=$ chest $x$-ray, $Q F T-I T=Q$ QuantiFERON ${ }^{\circledR}$-TB Gold In-Tube.

*51 of 126 cases were examined with this test.

${ }^{\dagger}$ Of the 48 culture-confirmed cases, 28 showed cultures positive in both sputum and bronchial washing specimens, 6 only in sputum and 14 only in bronchial washing specimens.

TB, 58 (79.5\%) were correctly excluded. HRCT findings in the diagnosis of PTB showed a significant difference between TB and non-TB patients $(\mathrm{P}<0.001)$ (data not shown). The most frequently observed CT findings (in order of decreasing frequency) were consolidation, mediastinal lymphadenopathy, tree-in-bud appearance, and nodules. Tree-in-bud nappearance was the only significant finding associated with active PTB status $(\mathrm{P}<0.001, \mathrm{OR}=5.10[2.37-10.99,95 \% \mathrm{CI}])$ (Table 3).

\section{Bronchoscopic characteristics in PTB patients}

Of the 54 patients confirmed with active PTB, 41 patients had TB and 13 patients did not have TB according to rapid diagnosis via bronchoscopic methods. As a result, two non-TB patients were misdiagnosed as having TB by bronchoscopic rapid diagnosis (Additional file 1: Table S1). Baseline characteristics were not different between TB and non-TB patients divided by bronchoscopic diagnosis (Additional file 1: Table S2).

The bronchoscopic findings are summarized in Table 4. Of the 54 PTB-confirmed patients, 42 patients had positive bronchial washing cultures (Table 4), 34 patients had positive sputum cultures, and 28 patients showed a positive result on both the sputum and bronchial washing cultures, but 14 patients had positive only in bronchial washing cultures (Additional file 1: Table S3).

Of the 54 PTB-confirmed patients, 41 (75.9\%) patients were rapidly diagnosed in less than 1 week by bronchial washing AFB smear, MTB-PCR and/or biopsy, and 13 patients were confirmed several weeks later by bronchial washing or sputum culture results. AFB smears produced by bronchial washing showed positive results for $25.9 \%$ (14/54) of the PTB-confirmed patients, and bronchoscopic biopsy results were positive in 22 of 23 patients who underwent a biopsy (Table 4). Of 23 patients who underwent a biopsy, a transbronchial biopsy was performed in 5 patients and a bronchial biopsy was performed in 18 patients with an endobronchial lesion (data not shown). MTB-PCR from a bronchial washing showed positive results in $61.1 \%(33 / 54)$ of patients (Table 4$)$, and 2 of the finally diagnosed 72 non-TB patients showed falsepositive results (Additional file 1: Table S1).

Table 2 First presenting symptoms of patients with sputum smear-negative TB

\begin{tabular}{|c|c|c|c|c|c|}
\hline Symptoms & $\begin{array}{c}\text { All suspects } \\
N=126\end{array}$ & $\begin{array}{c}\text { TB } \\
\mathrm{N}=54\end{array}$ & $\begin{array}{c}\text { Non-TB } \\
N=72\end{array}$ & P-value & OR $(95 \% \mathrm{Cl})$ \\
\hline Cough, n (\%) & $70(55.6)$ & $34(63.0)$ & $36(50.0)$ & 0.147 & $1.70(0.827-3.493)$ \\
\hline Sputum, n (\%) & $42(33.3)$ & $18(33.3)$ & $24(33.3)$ & 1.000 & $1.00(0.473-2.114)$ \\
\hline Fever, n (\%) & $27(21.4)$ & $13(24.1)$ & $14(19.4)$ & 0.531 & $1.31(0.559-3.087)$ \\
\hline Hemoptysis, n (\%) & $31(24.6)$ & $7(13.0)$ & $24(33.3)$ & 0.009 & $0.30(0.117-0.757)$ \\
\hline Chest wall pain, $\mathrm{n}(\%)$ & $22(17.5)$ & $13(24.1)$ & $9(12.5)$ & 0.090 & $2.22(0.870-5.662)$ \\
\hline Weight loss, n (\%) & $4(3.2)$ & $3(5.6)$ & $1(1.4)$ & 0.313 & $4.18(0.422-41.308)$ \\
\hline
\end{tabular}

Definition of abbreviations: $T B=$ tuberculosis, $n=$ number, $O R=$ odds ratio, $95 \% C l=95 \%$ confidence interval. 
Table 3 Multivariate regression analysis of CT findings in sputum smear-negative TB suspects

\begin{tabular}{lccccr}
\hline Characteristic & All suspects & TB & Non-TB & P-value & OR (95\% CI) \\
& $\mathbf{N}=\mathbf{1 2 6}$ & $\mathbf{N}=\mathbf{5 4}$ & $\mathbf{N = 7 2}$ & \\
\hline Consolidation, n (\%) & $88(69.8)$ & $38(70.4)$ & $50(69.4)$ & 0.726 & $1.05(0.484-2.257)$ \\
Nodule, n (\%) & $50(39.7)$ & $23(42.6)$ & $27(37.5)$ & 0.435 & $1.24(0.602-2.541)$ \\
Cavity, n (\%) & $23(18.3)$ & $15(27.8)$ & $8(11.1)$ & 0.050 & $3.08(1.195-7.924)$ \\
Tree-in-bud, n (\%) & $52(41.3)$ & $34(63.0)$ & $18(25.0)$ & $<0.001$ & $5.10(2.366-10.991)$ \\
Mediastinal LAP, n (\%) & $53(42.1)$ & $26(48.1)$ & $27(37.5)$ & 0.940 & $1.55(0.756-3.167)$ \\
\hline
\end{tabular}

Definition of abbreviations: $C T=$ computed tomography, $T B=$ tuberculosis, $n=$ number, $O R=$ odds ratio, $95 \% C l=95 \%$ confidence interval, $L A P=$ lymphadenopathy.

\section{Diagnostic accuracy of FOB and HRCT in sputum smear-} negative TB suspects

The diagnostic accuracy of FOB, HRCT, and the combination of FOB and HRCT in sputum smear-negative TB suspects is summarized in Table 5. The sensitivity of FOB in the rapid diagnosis of active PTB was $75.9 \%$ (95\% CI, 69.0-78.6\%), and the specificity was $97.2 \%$ (95\% CI, 92.0-99.2\%). The sensitivity of HRCT in the diagnosis of active PTB was 85.2\% (95\% CI, 75.2-92.3\%), and the specificity was $72.2 \%$ (95\% CI, 64.7-77.6\%). The PPV of FOB was $95.3 \%$ (95\% CI, 86.7-98.7\%) and the NPV of FOB was $84.3 \%$ (95\% CI, 79.8-86.1\%) in the rapid diagnosis of active PTB. These values were higher than the PPV of HRCT (69.7\% [95\% CI, 61.5-75.5\%]) and similar to the NPV of HRCT $(86.7 \%$ [95\% CI, 77.793.1\%]). The combination of FOB and HRCT improved the sensitivity to $96.3 \%$ and the NPV to $96.2 \%$.

\section{Discussion}

In this retrospective study, we found that FOB showed high sensitivity, specificity, PPV, and NPV in the rapid diagnosis of sputum smear-negative PTB, and the combination of FOB and HRCT increased the sensitivity and NPV.

Several previous studies evaluated clinical characteristics and scoring systems for the diagnosis of sputum smear-negative PTB [12-14]. Samb et al. [12] reported independent predictors of active PTB including a chronic cough lasting longer than 3 weeks, chest pains longer than 15 days, absence of sputum, and absence of dyspnea, and Lee et al. [14] reported that the lack of sputum was a positive predictor of active PTB. But even in these studies, the specificity of the clinical predictors or scoring system was low, and the PPV was reported at a mere $50 \%$. In our study, hemoptysis was the only negative predictor in sputum smear-negative TB suspects, and other clinical characteristics did not help in the prediction of active PTB.

We discovered that tree-in-bud appearance was a meaningful HRCT finding in the diagnosis of sputum smear-negative PTB. Recently, several studies have reported that tree-in-bud appearance, lobular consolidation and large nodules were positively associated with active PTB in sputum smear-negative TB suspects, but the diagnostic accuracy was not satisfactory $[14,15]$. Matsuoka et al. [16] stated that CT findings in sputum smear-negative patients differed from those in smearpositive patients and suggested that CT findings are not helpful in judging sputum smear-negative TB suspects.

Despite being less infectious than sputum smear-positive PTB, smear-negative PTB serves as an important cause of transmission in communities by delaying diagnosis and precluding initiation of treatment and often leads to complications of irreversible lung damage in infected individuals [4]. Therefore, sputum smear-negative PTB often requires more invasive diagnostic tools to be distinguished from other diseases such as lung cancer. In addition, in intermediate or high TB burden countries such as South Korea, beginning unnecessary anti-TB treatment prior to

Table 4 Bronchoscopic findings in $\mathbf{5 4}$ patients confirmed to have active PTB

\begin{tabular}{|c|c|c|c|}
\hline & $\begin{array}{c}\text { TB } \\
N=54\end{array}$ & $\begin{array}{l}\text { Culture confirmed } \\
\qquad N=48\end{array}$ & $\begin{array}{l}\text { Biopsy confirmed } \\
\qquad N=6\end{array}$ \\
\hline Bronchial washing culture & $42 / 54(77.8)$ & $42 / 48(87.5)$ & $0 / 6(0.0)$ \\
\hline Bronchial washing AFB smear & $14 / 54(25.9)$ & $12 / 48(25.0)$ & $2 / 6(33.3)$ \\
\hline Bronchial washing MTB-PCR & $33 / 54(61.1)$ & $32 / 48(66.7)$ & $1 / 6(16.7)$ \\
\hline Bronchoscopic biopsy (TBB + bronchial biopsy) & $22 / 23(95.7)$ & $16 / 17(94.1)$ & $6 / 6(100.0)$ \\
\hline Bronchial washing AFB smear + MTB-PCR + bronchoscopic biopsy & $41 / 54(75.9)$ & $35 / 48(72.9)$ & $6 / 6(100.0)$ \\
\hline
\end{tabular}

Definition of abbreviations: $P T B=$ pulmonary tuberculosis, $T B=$ tuberculosis, $A F B=$ acid-fast bacilli, $M T B=$ mycobacterial tuberculosis, $P C R=$ polymerase chain. reaction, $T B B=$ transbronchial lung biopsy.

${ }^{*}$ Numbers of patients with positive results in each test/total numbers of patients who underwent each test (\%). 
Table 5 Diagnostic accuracy in all sputum smear-negative TB suspects

\begin{tabular}{lcccccc}
\hline & Sensitivity (95\% Cl) & Specificity (95\% Cl) & PPV (95\% Cl) & NPV (95\% Cl) & +LR (95\% Cl) & -LR (95\% Cl) \\
\hline FOB & $0.759(0.690-0.786)$ & $0.972(0.920-0.992)$ & $0.953(0.867-0.987)$ & $0.843(0.798-0.861)$ & $27.333(8.674-99.257)$ & $0.248(0.216-0.337)$ \\
CT & $0.852(0.752-0.923)$ & $0.722(0.647-0.776)$ & $0.697(0.615-0.755)$ & $0.867(0.777-0.931)$ & $3.067(2.131-4.117)$ & $0.205(0.099-0.383)$ \\
FOB + CT & $0.963(0.879-0.993)$ & $0.708(0.645-0.731)$ & $0.712(0.650-0.735)$ & $0.962(0.876-0.993)$ & $3.302(2.477-3.696)$ & $0.052(0.009-0.188)$ \\
\hline
\end{tabular}

Definition of abbreviations: $T B=$ tuberculosis, $P P V=$ positive predictive value, $N P V=$ negative predictive value, $+L R=$ positive likelihood ratio, $-L R=$ negative likelihood ratio, $95 \% C l=95 \%$ confidence interval, $F O B=$ fiberoptic bronchoscopy, $C T=$ computed tomography.

receiving results of mycobacterial culture may cause unnecessary economic burden to the community and drug side effects to the patient.

Lee et al. [14] reported that the sensitivity and the specificity of sputum TB-PCR was $43.2 \%$ (95\% CI, 27-60\%) and $97.7 \%$ (95\% CI, 86-99\%), respectively. Because of its low sensitivity and NPV (66.7\% [95\% CI, 54-78\%]), sputum TB-PCR alone was limited in ruling out TB. In a recent meta-analysis study [17], the sensitivity and the specificity of QFT-IT was 80\% (95\% CI, 75-84\%) and 79\% (95\% CI, 78-84\%), respectively, and the sensitivity and the specificity of T-SPOT TB was $81 \%$ (95\% CI, 78-84\%) and 59\% (95\% CI, 56-62\%), respectively. The diagnostic sensitivity of both interferon-gamma release assays (IGRAs) was higher than that of the TST, but the sensitivity of IGRAs was still not high enough for IGRAs to be used as a rule out test for TB. In addition, the specificity of the IGRAs was not sufficient for the diagnosis of active TB disease with differentiation from latent TB infection.

In previous studies, FOB had a sensitivity of $80-93 \%$ and a specificity of $70-95 \%[8,9,18,19]$ for rapid diagnosis of sputum smear-negative PTB, and HRCT had a sensitivity of $60-80 \%$ and a specificity of $50-70 \%$ [14, 20,21]. Our results indicate that HRCT has a similar sensitivity of $85.2 \%$ and specificity of $72.2 \%$, but HRCT alone is limited as usual in the diagnosis of PTB in sputum smear-negative TB suspects due to its low PPV of $69.7 \%$ (positive likelihood ratio $(\mathrm{LR}+), 3.067$ [95\% CI, 2.131-4.117]). We found that FOB alone is useful for the rapid diagnosis of sputum smearnegative PTB with a high PPV of 95.3\% ( $\mathrm{LR}+=27.333$ [95\% CI, 8.674-99.257]). FOB is also useful for the exclusion of non-TB cases from patients suspected of having active PTB with a high specificity and NPV in our setting. The combination of FOB with HRCT increased the sensitivity to $96.3 \%$ and NPV to $96.2 \%$.

The high cost and concerns about the invasiveness of FOB and radiation exposure of HRCT limit the usefulness of these tests, but FOB is a safe and widely performed procedure. Previous studies [22,23] have reported that with the help of oxygenation, adequate premedication, and performance by experienced physicians, FOB shows very low complication rates and few life-threatening side effects. One previous study investigating the cost-effectiveness of FOB and HRCT in the diagnosis of PTB [21] reported that FOB and HRCT play significant roles in the moderate or high PTB probability setting compared with the low PTB probability setting. Considering the high specificity of our study, FOB and/ or HRCT would be useful in a high TB burden country as well as in an intermediate TB burden country such as South Korea.

There are several limitations in this study. First, it was a retrospective study and the study population and clinical setting were selective and limited, so it is difficult to generalize this result to other settings. Second, the commonly used TST was not evaluated in our study. This was based on previous studies showing the limitation of TST for evaluating TB in South Korea due to BCG vaccination $[24,25]$. Third, the use of FOB in the diagnosis of PTB is not clinically available worldwide. To improve diagnostic accuracy and ensure safety, a well-trained pulmonologist is essential. High cost and the complication risk limit the use of FOB in other situations. However, our study demonstrates that FOB and FOB with HRCT can play an important role in the rapid diagnosis of active PTB in sputum smear-negative patients suspected of TB.

\section{Conclusions}

In conclusion, our results suggest that FOB has good sensitivity, specificity, PPV, and NPV and was useful in the rapid diagnosis of sputum smear-negative PTB and the exclusion of non-TB in sputum smear-negative TB-suspected patients. HRCT alone was limited for the diagnosis of active PTB, but the combination of FOB and HRCT may improve the sensitivity of FOB in the rapid diagnosis of sputum smear-negative PTB. Based on our results, we suggest that physicians actively consider performing FOB and HRCT in sputum smear-negative patients suspected of TB.

\section{Additional file}

\footnotetext{
Additional file 1: Tables S1-S3. Bronchoscopic findings of all subjects. Tables S2. Diagnostic and clinical characteristics of active PTB confirmed 54 patients according to bronchoscopic diagnosis. Tables S3. Bronchoscopic findings in 54 active PTB patients according to sputum mycobacteria culture results.
}

\section{Abbreviations}

AFB: Acid-fast bacilli; BCG: Bacillus Calmette-Geurin; FOB: Fiberoptic bronchoscopy; HRCT: High-resolution computed tomography; IGRAs: Interferongamma release assays; LTBI: Latent TB infection; MTB: Mycobacterium tuberculosis; NPV: Negative predictive value; NTM: Non-tuberculous 
mycobacteria; PCR: Polymerase chain reaction; PPV: Positive predictive value; PTB: Pulmonary tuberculosis; QFT-IT: QuantiFERON ${ }^{\circledR}$-TB Gold In-Tube; TB: Tuberculosis; TST: Tuberculin skin test.

\section{Competing interests}

The author(s) declare that they have no competing interests.

\section{Authors' contributions}

All authors have read and approved the final manuscript. SJA and BMK designed and conducted the study; collected, analyzed, and interpreted the data; and wrote the manuscript. KTH, CYS, KHJ, and ACM analyzed and interpreted the data and contributed to the writing of the manuscript. All authors read and approved the final manuscript.

\section{Acknowledgements}

The authors are grateful for statistical support provided by the medical research supporting section of the Yonsei University College of Medicine.

\section{Author details}

'Department of Internal Medicine, Yonsei University College of Medicine, Gangnam Severance Hospital, 211 Eonju-ro, Gangnam-gu Seoul 135-720, South Korea. ${ }^{2}$ Department of Radiology, Yonsei University College of Medicine, Gangnam Severance Hospital, 211 Eonju-ro, Gangnam-gu, 211 Eonju-ro, Gangnam-gu, Seoul 135-720 135-720, South Korea.

Received: 13 December 2011 Accepted: 14 June 2012

Published: 22 June 2012

\section{References}

1. World Health Organization (WHO): Global tuberculosis control. 2011, http://www.who.int/tb/publications/global_report/en/index.html.

2. Nair N, Wares F, Sahu S: Tuberculosis in the WHO South-East Asia Region. Bull World Health Organ 2010, 88:164.

3. Korean National Tuberculosis Association (KNTA): Trend of case notification rate per 100,000 by year, 2004-2010. https://www.knta.or.kr/inform/ sub 03 10.asp.

4. Foulds J, O'Brien R: New tools for the diagnosis of tuberculosis: the perspective of developing countries. Int J Tuberc Lung Dis 1998, 2:778-783.

5. Tozkoparan E, Deniz O, Ciftci F, Bozkanat E, Bicak M, Mutlu H, Ors F, Bilgic H, Demirci N: The roles of HRCT and clinical parameters in assessing activity of suspected smearnegative pulmonary tuberculosis. Arch Med Respir 2005, 36:166-170.

6. Kanaya AM, Glidden DV, Chambers HF: Identifying pulmonary tuberculosis in patients with negative sputum smear results. Chest 2001, 120:349-355.

7. Richeldi L: An Update on the Diagnosis of Tuberculosis Infection. Am J Respir Crit Care Med 2006, 174:736-742.

8. Charoenratanakul S, Dejsomritrutai W, Chaiprasert A: Diagnostic role of fiberoptic bronchoscopy in suspected smear negative pulmonary tuberculosis. Respir Med 1995, 89:621-623.

9. Willcox PA, Benatar SR, Potgieter PD: Use of the flexible fibreoptic bronchoscope in diagnosis of sputum-negative pulmonary tuberculosis. Thorax 1982, 37:598-601.

10. Altaf Bachh A, Gupta R, Haq I, Varudkar HG: Diagnosing sputum/smearnegative pulmonary tuberculosis: Does fibre-optic bronchoscopy play a significant role? Lung India 2010, 27:58-62.

11. Araz O, Akgun M, Saglam L, Ozden K, Mirici A: The diagnostic value of bronchoscopy in smear negative cases with pulmonary tuberculosis. Tuberk Toraks 2008, 56:150-157.

12. Samb B, Henzel D, Daley CL, Mugusi F, Niyongabo T, MlikaCabanne N, Kamanfu G, Aubry P, Mbaga I, Larouze B, Murray JF: Methods for diagnosing tuberculosis among in-patients in Eastern Africa whose sputum smears are negative. Int I Tuberc Lung Dis 1997, 1:25-30.

13. Tessema TA, Bjune G, Assefa G, Bjorvat B: An evaluation of the diagnostic value of clinical and radiological manifestations in patients attending the addis ababa tuberculosis centre. Scand J Infect Dis 2001, 33:355-361.

14. Lee HM, Shin JW, Kim JY, Park IW, Choi BW, Choi JC, Seo JS, Kim CW: HRCT and whole-blood interferon-gamma assay for the rapid diagnosis of smear-negative pulmonary tuberculosis. Respiration 2010, 79:454-460.

15. Nakanishi M, Demura Y, Ameshima S, Kosaka N, Chiba Y, Nishikawa S, Itoh $H$, Ishizaki T: Utility of high-resolution computed tomography for predicting risk of sputum smear-negative pulmonary tuberculosis. Eur J Radiol 2010, 73:545-550.

16. Matsuoka S, Uchiyama K, Shima H, Suzuki K, Shimura A, Sasaki Y, Yamagishi F: Relationship between $C T$ findings of pulmonary tuberculosis and the number of acid-fast bacilli on sputum smears. Clin Imaging 2004, 28:119-123.

17. Sester M, Sotgiu G, Lange C, Giehl C, Girardi E, Migliori GB, Bossink A, Dheda K, Diel $R$, Dominguez J, et al: Interferon-gamma release assays for the diagnosis of active tuberculosis: a systematic review and meta-analysis. Eur Respir J 2011, 37:100-111.

18. Liam CK, Chen YC, Yap SF, Srinivas P, Poi PJ: Detection of Mycobacterium tuberculosis in bronchoalveolar lavage from patients with sputum smear-negative pulmonary tuberculosis using a polymerase chain reaction assay. Respirology 1998, 3:125-129.

19. Wong CF, Yew WW, Chan CY, Au LY, Cheung SW, Cheng AF: Rapid diagnosis of smear-negative pulmonary tuberculosis via fibreoptic bronchoscopy: utility of polymerase chain reaction in bronchial aspirates as an adjunct to transbronchial biopsies. Respir Med 1998, 92:815-819.

20. Lai FM, Liam CK, Paramsothy M, George J: The role of 67gallium scintigraphy and high resolution computed tomography as predictors of disease activity in sputum smear-negative pulmonary tuberculosis. Int J Tuberc Lung Dis 1997, 1:563-569.

21. Lim TK, Cherian J, Poh KL, Leong TY: The rapid diagnosis of smearnegative pulmonary tuberculosis: a cost-effectiveness analysis. Respirology 2000, 5:403-409.

22. Credle WF Jr, Smiddy JF, Elliott RC: Complications of fiberoptic bronchoscopy. Am Rev Respir Dis 1974, 109:67-72.

23. Jolliet $\mathrm{P}$, Chevrolet JC: Bronchoscopy in the intensive care unit. Intensive Care Med 1992, 18:160-169.

24. Choi JC, Shin JW, Kim JY, Park IW, Choi BW, Lee MK: The effect of previous tuberculin skin test on the follow-up examination of whole-blood interferon-gamma assay in the screening for latent tuberculosis infection. Chest 2008, 133:1415-1420.

25. Kang YA, Lee HW, Yoon HI, Cho B, Han SK, Shim YS, Yim JJ: Discrepancy between the tuberculin skin test and the whole-blood interferon gamma assay for the diagnosis of latent tuberculosis infection in an intermediate tuberculosis-burden country. JAMA 2005, 293:2756-2761.

doi:10.1186/1471-2334-12-141

Cite this article as: Shin et al:: Fiberoptic bronchoscopy for the rapid diagnosis of smear-negative pulmonary tuberculosis. BMC Infectious Diseases 2012 12:141.

\section{Submit your next manuscript to BioMed Central and take full advantage of:}

- Convenient online submission

- Thorough peer review

- No space constraints or color figure charges

- Immediate publication on acceptance

- Inclusion in PubMed, CAS, Scopus and Google Scholar

- Research which is freely available for redistribution 